\title{
Effect of butylated hydroxytoluene on cryopreservation of Boer goat semen in Tris egg yolk extender.
}

\begin{abstract}
The aim of this study was to determine the effect of butylated hydroxytoluene (BHT), a lipidsoluble anti-oxidant added in different concentrations to the Tris egg yolk extenders on semen cytological parameters pre freezing and post thawing (motility, morphology, viability, acrosome integrity and membrane integrity) of Boer goat spermatozoa. A total of 40 ejaculates from four Boer goat bucks were collected using an artificial vagina. Ten replicates of the ejaculates were diluted with a Tris egg yolk based extender which contained various concentrations $(0.5 \mathrm{mM}, 1.0 \mathrm{mM}, 2.0 \mathrm{mM}$ and $3.0 \mathrm{mM})$ of butylated hydroxytoluene while one sample was processed without supplementation of antioxidant and served as control. The diluted semen was cooled at $4{ }^{\circ} \mathrm{C}$ and loaded into the straw and then stored in liquid nitrogen. It was evident that supplementation of BHT produces positive effect in terms of motility, membrane integrity and acrosome integrity in comparison with the control group in cooled and frozen Boer goat semen. Results showed significant differences in motility, membrane integrity, acrosome integrity and viability of cooled and frozen Boer goat spermatozoa at different concentrations. Motility, membrane integrity, acrosome integrity and viability was significantly higher in all treated groups than the control group $(\mathrm{P}<0.05)$ while there was no significant differences $(\mathrm{P}>0.05)$ in morphology trait between all group in cooled semen. However, improvement $(\mathrm{P}<0.05)$ was observed only in terms of the membrane integrity and acrosome integrity compared to the control and other treated groups in frozen semen. In conclusion, BHT can be used in cryopreservation of Boer goat semen in order to reduce the oxidative stress on spermatozoa.
\end{abstract}

Keyword: Butylated hydroxytoluene; Goat; Semen; Freezing; Extenders; Antioxidants. 